\title{
A HERMITE-TYPE ADAPTIVE SEMI-LAGRANGIAN SCHEME
}

\author{
MICHEL MEHRENBERGER *, ERIC VIOLARD ** \\ * IRMA (CNRS UMR-7005), Université Louis Pasteur \\ 7, rue René Descartes, 67084 Strasbourg, France \\ e-mail: mehrenbe@math.u-strasbg.fr \\ ** Laboratoire ICPS - LSIIT (CNRS UMR-7005), Université Louis Pasteur \\ Pôle API, Boulevard Sébastien Brant, 67400 Illkirch, France \\ e-mail:violard@icps.u-strasbg.fr
}

\begin{abstract}
We study a new Hermite-type interpolating operator arising in a semi-Lagrangian scheme for solving the Vlasov equation in the 2D phase space. Numerical results on uniform and adaptive grids are shown and compared with the biquadratic Lagrange interpolation introduced in (Campos Pinto and Mehrenberger, 2004) in the case of a rotating Gaussian.
\end{abstract}

Keywords: adaptive method, Vlasov equation, numerical simulation, Hermite operator

\section{Introduction}

Adaptive semi-Lagrangian schemes for solving the Vlasov equation in the phase space have recently been developed. They include wavelet techniques (Gutnic et al., 2004; Gutnic et al., 2005), the moving mesh method (Sonnendrücker et al., 2004), and hierarchical finite element decomposition (Campos Pinto and Mehrenberger, 2004; Campos Pinto and Mehrenberger, 2005). One main advantage of the latter method is that the underlying dyadic partition of cells allows for an efficient parallelization. It has been implemented with a biquadratic Lagrange interpolation. But the use of higher-order methods is not straightforward in that context. The same problem in fact occurs in the case of semi-Lagrangian schemes on unstructured grids. One solution there was to use a Hermitetype interpolation (see (Besse and Sonnendrücker, 2003) and also (Nakamura and Yabe, 1999)). We propose here to do the same in the adaptive context. Thanks to a well chosen Hermite interpolation recently found (Hong and Schumaker, 2004), we thus obtain a more accurate scheme.

The paper is organized as follows: Section 2 presents two interpolating operators that we designed for our numerical scheme. First, we recall the Lagrange operator and then we present the new Hermite one. Section 3 briefly recalls our uniform and adaptive semi-Lagrangian schemes. Section 4 focuses on the crucial point of the computational cost of the two operators. We propose ef- ficient algorithms to compute the interpolated value as a sequence of assignments. Section 5 completes the definition of our adaptive scheme. For each operator, criteria for compressing cells of the adaptive mesh are provided. Finally, Section 6 shows our experimental results before concluding.

\section{Local Interpolating Operators}

Notation. We use the square $\Omega=\left[0,1\left[^{2}\right.\right.$ as a computational domain. It is decomposed using a partition $\mathcal{M}$ of square shaped cells $\alpha=\left[k 2^{-j},(k+1) 2^{-j}\left[\times\left[\ell 2^{-j},(\ell+\right.\right.\right.$ 1) $2^{-j}$ [, where $k, \ell$ and $j$ are integers, and $j$ denotes the level of the cell. For a point $(x, y) \in \Omega$, we can thus define a unique cell $\alpha_{x, y} \in \mathcal{M}$ such that $(x, y) \in \alpha_{x, y}$.

Given a cell $\alpha$, we denote by $(0,0)_{\alpha},(1,0)_{\alpha},(1,1)_{\alpha}$, $(0,1)_{\alpha}$ its four corners and, more generally, $(\lambda, \mu)_{\alpha}$ will be the point whose local coordinates in $\alpha$ are $\lambda, \mu \in$ $[0,1]^{2}$. Let $T_{k}^{\alpha}, k=0, \ldots, 3$ be the four triangles obtained by subdividing the cell $\alpha$ with the diagonals (Fig. 1).

For $d \in \mathbb{N}$, we classically define

$$
Q_{d}=\left\{\sum a_{i, j} x^{i} y^{j}, \quad i, j \leq d\right\},
$$

and

$$
P_{d}=\left\{\sum a_{i, j} x^{i} y^{j}, \quad i+j \leq d\right\}
$$

Now, let $f$ be a function defined on $\Omega$ extended to $\mathbb{R}^{2}$ by zero (we can similarly extend it by periodicity), and $(x, y) \in \Omega$. 


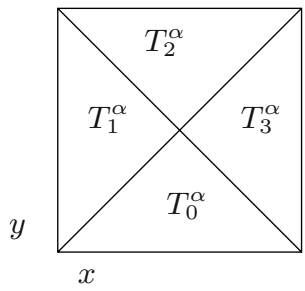

Fig. 1. Four triangles of a cell $\alpha$.

Biquadratic Lagrange interpolation. $P_{L} f(x, y)$ is defined as the unique element of $Q_{2}$ on the cell $\alpha=\alpha_{x, y}$ such that it coincides with $f$ on the nine degrees of freedom at the equidistant nodes:

$$
P_{L} f\left((p, q)_{\alpha}\right)=f\left((p, q)_{\alpha}\right) \quad p, q=0,1 / 2,1 .
$$

Hermite interpolation. Assume that $f$ is differentiable on each cell $\alpha . P_{H} f(x, y)$ is then the unique $C^{1}$ spline on the cell $\alpha=\alpha_{x, y}, P_{3}$ on the triangles $T_{k}^{\alpha}, k=0, \ldots, 3$ such that there is coincidence on the twelve degrees of freedom at the corners:

$$
f(a), \partial_{x} f(a), \partial_{y} f(a), \quad a=(p, q)_{\alpha} \quad p, q=0,1,
$$

and also on the four normal derivatives on the edges, i.e.,

$$
\partial_{y} f\left((1 / 2, p)_{\alpha}\right), \partial_{x} f\left((p, 1 / 2)_{\alpha}\right), \quad p=0,1 .
$$

\section{General Iterative Scheme}

A semi-Lagrangian scheme takes the form of a succession of interpolation and transport steps.

Let us consider the Vlasov equation

$$
\partial_{t} f+y \partial_{x} f+E(t, x) \partial_{y} f=0 .
$$

The electric field $E$ is generally computed via the Poisson equation. We will suppose here that it is a known function. We define classically the characteristic curves

$$
Z(t ; s, x, y)=(X, Y)=(X(t ; s, x, y), Y(t ; s, x, y)),
$$

such that

$$
\frac{\mathrm{d}}{\mathrm{d} t} X=y, \quad \frac{\mathrm{d}}{\mathrm{d} t} Y=E
$$

together with $Z(s ; s, x, y)=(x, y)$. The function $f=$ $f(t, x, y)$ that we want to approximate, satisfying (1), is constant along these characteristics:

$$
f(t, x, y)=f(t, Z(t ; t, x, y))=f_{0}(Z(0 ; t, x, y)),
$$

where $f_{0}$ is a given initial condition defined in $\Omega$ and complemented by zero outside $\Omega$.

Let $\Delta t>0$ be the time step, and $\mathcal{T}^{n}$ be the exact backward transport operator at iteration step $n$, which is defined by

$$
\mathcal{T}^{n}(x, y)=Z((n-1) \Delta t ; n \Delta t, x, y) .
$$

Since $f$ is constant along the characteristics, we have

$$
f((n+1) \Delta t, x, y)=f\left(n \Delta t, \mathcal{T}^{n+1}(x, y)\right) .
$$

We focus here on the errors produced by the interpolating process and we shall assume that the exact transport operator (and thus also the exact solution) is known. In the general case, we should use an approximation of the exact transport operator.

The iterative scheme then consists in finding the degrees of freedom at each iteration step $n$, which give a representation $f^{n}$, complemented by zero outside $\Omega$. We fix a resolution level $J \in \mathbb{N}^{*}$.

Uniform scheme. The semi-Lagrangian method with the propagation of the gradients was studied on uniform grids in (Besse and Sonnendrücker, 2003; Nakamura and Yabe, 1999). We consider a uniform grid $\mathcal{M}_{J}$ of $2^{2 J}$ cells.

- (Iteration step $n=0$ ) We compute the degrees of freedom from $f_{0}$ on the corresponding grid, which gives a representation $f^{0}$ at iteration step 0 .

- (Iteration step $n+1)$ For each point $(x, y)$ corresponding to a degree of freedom, we compute the backward advected point $\mathcal{T}^{n+1}(x, y)$. The new value of the degree of freedom is thus $f^{n}\left(\mathcal{T}^{n+1}(x, y)\right.$ ) (or $\frac{\mathrm{d}}{\mathrm{d} z} f^{n}\left(\mathcal{T}^{n+1}(x, y)\right)$, with $z=x$ or $\left.y\right)$. We thus have a representation $f^{n+1}$ at iteration step $n+1$.

Adaptive scheme. We will use a compression step $(\tilde{f}, \tilde{\mathcal{M}})=\mathcal{C}(f, \mathcal{M})$. From a representation of a function $f$ on a mesh $\mathcal{M}$, we will derive a new coarser representation $\tilde{f}$ on a mesh $\tilde{\mathcal{M}}$. Note that a cell $\alpha_{k, \ell}^{j}$ is partitioned into four cells $\alpha_{2 k+k^{\prime}, 2 \ell+\ell^{\prime}}^{j+1}, k^{\prime}, \ell^{\prime}=0,1$. We will say that the cell $\alpha_{k, \ell}^{j}$ is the mother cell of the four daughter cells $\alpha_{2 k+k^{\prime}, 2 \ell+\ell^{\prime}}^{j+1}, k^{\prime}, \ell^{\prime}=0,1$. The compression can then be done locally, by comparing the representation of the current function on the four daughters cells with the interpolated function on the mother cell. If the two representations are not far from each other, we will keep the coarser representation on the mother cell. Specific tests for the biquadratic Lagrange interpolation and for the Hermite interpolation will be defined.

We also define a prediction step $\tilde{\mathcal{M}}_{n}=\mathcal{T}^{n+1}\left(\mathcal{M}_{n}\right)$. From a mesh $\mathcal{M}_{n}$ and the backward advection operator $\mathcal{T}^{n+1}$, we compute a new mesh $\tilde{\mathcal{M}}_{n}$. This is performed by beginning with a coarse mesh and recursively refining each cell of level $j \leq J$, if the backward advected center of the cell falls on a cell of $\mathcal{M}^{n}$ whose level is less than $j$.

The algorithm is then as follows:

- (Iteration step $n=0$ ) From $f_{u}^{0}$ obtained by the uniform algorithm, we compress the solution and obtain the representation $\left(f^{0}, \mathcal{M}^{0}\right)=\mathcal{C}\left(\mathcal{M}_{J}, f_{u}^{0}\right)$. 
- (Iteration step $n+1$ ) We predict a first grid $\mathcal{T}^{n+1}\left(\mathcal{M}^{n}\right)$ from $\mathcal{M}^{n}$, compute $f_{1}^{n+1}$ as in the uniform algorithm (replacing $\mathcal{M}_{J}$ with $\mathcal{T}^{n+1}\left(\mathcal{M}^{n}\right)$ ), and next compute the representation of $f$ on $\mathcal{M}^{n+1}$ : $\left(f^{n+1}, \mathcal{M}^{n+1}\right)=\mathcal{C}\left(\mathcal{T}^{n+1}\left(\mathcal{M}^{n}\right), f_{1}^{n+1}\right)$.

\section{Fast Formulas}

Our formulas are defined on any square cell $\alpha=[a, a+$ $h[\times[b, b+h[$, i.e., for $a \leq x<a+h$ and $b \leq y<b+h$.

Biquadratic Lagrange interpolation. Given nine numbers $g_{i, j}, i, j=0,1,2$, the function $g(x, y)$ of a degree no greater than 2 that satisfies

$$
g(a+i h / 2, b+j h / 2)=g_{i, j}, \quad \text { for } i, j=0,1,2,
$$

is uniquely determined. We can compute it as follows: We first set $N=1 / h$ (which can be precomputed) and $t_{0}=(x-a) N, \quad t_{1}=2 t_{0}-1$, which gives

$$
\begin{aligned}
& h_{0}=g_{1, j}+t_{1}\left(g_{1, j}-g_{0, j}\right), \\
& h_{1}=g_{1, j}+t_{1}\left(g_{2, j}-g_{1, j}\right), \\
& g_{j}=h_{0}+t_{0}\left(h_{1}-h_{0}\right) \text { for } j=0,1,2 .
\end{aligned}
$$

We next set $t_{0}=(v-b) N, \quad t_{1}=2 t_{0}-1$, so that we have

$$
\begin{aligned}
h_{0} & =g_{1}+t_{1}\left(g_{1}-g_{0}\right), \\
h_{1} & =g_{1}+t_{1}\left(g_{2}-g_{1}\right), \\
g(x, v) & =h_{0}+t_{0}\left(h_{1}-h_{0}\right) .
\end{aligned}
$$

This procedure thus needs only 10 assignments, 16 multiplications and 28 additions (or subtractions).

Hermite interpolation. Given 16 numbers $g_{i, j}, g_{i, j}^{x}$, $g_{i, j}^{y}, i, j=0,1, g_{0}^{x}, g_{1}^{x}, g_{0}^{y}$ and $g_{1}^{y}$, the $C^{1}$ cubic spline $P_{3}$ on the triangles $T_{0}^{\alpha}, T_{1}^{\alpha}, T_{2}^{\alpha}$ and $T_{3}^{\alpha}$, satisfying

$$
\begin{aligned}
g(a+i h, b+j h) & =g_{i, j}, \\
\partial_{x} g(a+i h, b+j h) & =g_{i, j}^{x}, \\
\partial_{y} g(a+i h, b+j h) & =g_{i, j}^{y} \text { for } i, j=0,1
\end{aligned}
$$

and

$$
\begin{aligned}
& \partial_{x} g(a+i h, b+h / 2)=g_{i}^{x}, \\
& \partial_{y} g(a+h / 2, b+i h)=g_{i}^{y} \text { for } i=0,1,
\end{aligned}
$$

is uniquely determined.

On the triangle $T_{0}^{\alpha}$ we can compute it as follows (formulas on the other triangles can be similarly derived): By setting $u=(x-a) N$ and $v=(y-b) N$, we obtain

$$
\begin{aligned}
& g(x, y) \\
& \quad=g_{00}+\left(2 u^{3}-3 u^{2}\right)\left(g_{00}-g_{10}\right) \\
& \quad+\left(v^{3}-3 v^{2}+3 u v^{2}\right)\left(g_{00}-g_{01}\right) \\
& \quad+\left(v^{3}-3 u v^{2}\right)\left(g_{10}-g_{11}\right) \\
& \quad+h\left(\left(2 / 3 v^{3}-2 u^{2}-3 / 2 v^{2}+u+3 / 2 u v^{2}+u^{3}\right) g_{00}^{u}\right.
\end{aligned}
$$

$$
\begin{aligned}
& +\left(-1 / 2 v^{2}+u v^{2}+v-3 u v+1 / 6 v^{3}+2 u^{2} v\right) g_{00}^{v} \\
& +\left(2 / 3 v^{3}-2 v^{2}+4\left(-u^{2}+u\right) v\right) g_{0}^{v} \\
& +\left(-2 / 3 v^{3}-u^{2}+3 / 2 u v^{2}+u^{3}\right) g_{10}^{u} \\
& +\left(1 / 2 v^{2}-u v^{2}-u v+1 / 6 v^{3}+2 u^{2} v\right) g_{10}^{v} \\
& +\left(4 / 3 v^{3}-2 u v^{2}\right) g_{1}^{u}+\left(-2 / 3 v^{3}+1 / 2 u v^{2}\right) g_{11}^{u} \\
& +\left(5 / 6 v^{3}-u v^{2}\right) g_{11}^{v}-2 / 3 v^{3} g_{1}^{v} \\
& +\left(2 / 3 v^{3}+1 / 2\left(-v^{2}+u v^{2}\right)\right) g_{01}^{u} \\
& +\left(5 / 6 v^{3}-v^{2}+u v^{2}\right) g_{01}^{v} \\
& \left.+\left(-4 / 3 v^{3}+2\left(v^{2}-u v^{2}\right)\right) g_{0}^{u}\right)
\end{aligned}
$$

We used the 'optimize' function of the 'codegen' package of Maple to reduce the cost of these interpolation operators and implement them in our code. We applied this optimization in the practical case where $a, b=0$ and $h$ is the size of a mesh cell.

For example, in our code, the cost of the computation of $g(x, y)$ on the triangle $T_{0}^{\alpha}$ by the Hermite operator is 18 assignments, 49 multiplications and 53 additions. It is obtained by introducing some auxiliary variables as follows:

$$
\begin{array}{rll}
v_{1}=v^{2}, & v_{2}=3 v_{1}, & v_{3}=u v_{2}, \\
v_{4}=v v_{1}, & v_{5}=2 / 3 v_{4}, & v_{6}=4 / 3 v_{4}, \\
v_{7}=5 / 6 v_{4}, & v_{8}=u v_{1}, & v_{9}=1 / 6 v_{4}+2 x_{1} v, \\
x_{1}=u^{2}, & x_{2}=u u_{1}, \quad x_{3}=u x_{2}, \quad x_{4}=x_{3}+3 / 2 v_{8} .
\end{array}
$$

Moreover, for the Hermite operator, instead of computing $g(x, y), \partial_{x} g(x, y)$ and $\partial_{v} g(x, y)$ separately, we compute them together, which reduces the number of required elementary operations and reduces the computation cost at least by $10 \%$ for most processor architectures.

\section{Compression Formulas}

Hermite compression. In the case of the Hermite interpolation, the compression test used for the four daughter cells of a given cell $\alpha$ of length $h$ is

$$
d_{0}^{x}+d_{1}^{x}+d_{0}^{v}+d_{1}^{v} \leq \varepsilon,
$$

with $d_{k}^{x}=\left|f\left((1 / 2, k)_{\alpha}\right)-\tilde{f}_{1 / 2 k}\right|$, where

$$
\begin{aligned}
\tilde{f}_{1 / 2 k}=\left(f\left((0, k)_{\alpha}\right)+f\left((1, k)_{\alpha}\right)\right) / 2 \\
+h / 8\left(\partial_{x} f\left((0, k)_{\alpha}\right)+\partial_{x} f\left((1, k)_{\alpha}\right)\right)
\end{aligned}
$$

and $d_{k}^{v}=\left|f\left((k, 1 / 2)_{\alpha}-\tilde{f}_{k 1 / 2}\right)\right|$, where

$$
\begin{aligned}
\tilde{f}_{k 1 / 2}=((f( & \left.\left.(k, 0)_{\alpha}\right)+f\left((k, 1)_{\alpha}\right)\right) / 2 \\
& +h / 8\left(\partial_{v} f\left((k, 0)_{\alpha}\right)+\partial_{v} f\left((k, 1)_{\alpha}\right)\right)
\end{aligned}
$$

for $k=0,1$. Here $\tilde{f}_{k 1 / 2}$ and $\tilde{f}_{1 / 2 k}$ are the reconstructed values at the middle of the edges, with the 1D Hermite interpolation operator. Note that this compression test is 
not exactly the same as that introduced in Section 3. However, it provides an easy and cheap local criterion for the order of accuracy, because it only uses a $1 D$ interpolation, and it has been successfully applied in our testcase. Other criteria may be used and compared.

Biquadratic Lagrange compression. For a given mother cell $\alpha$, the compression test used is

$$
\sum_{p, q=0}^{4}\left|f\left((p / 4, q / 4)_{\alpha}\right)-\tilde{f}_{p q}\right| \leq \varepsilon
$$

where $\tilde{f}_{p q}$ is the value obtained by interpolation on the cell $\alpha$ at point $(p / 4, q / 4)_{\alpha}$.

\section{Numerical Results}

For initial data we take

$$
f_{0}(x, y)=e^{-0.07\left((40(x-0.5)+4.8)^{2}+(40(y-0.5)+4.8)^{2}\right)},
$$

in $\Omega=[0,1]^{2}$ and $\Delta t=0.19635$. The transport operator is given here by a rotation of angle $\Delta t$ around the center $(0.5,0.5)$, so that the equation that is numerically solved is (1) with $E(t, x)=-(x-0.5)$.

We implemented our schemes in $\mathrm{C}++$ and executed our code on a Pentium 4 processor $(3.06 \mathrm{GHz}, 512 \mathrm{MB}$ RAM). We considered our adaptive scheme for $\epsilon=10^{-4}$, $10^{-5}, 10^{-6}, 10^{-7}$ and $10^{-8}$. The error is computed on a uniform grid of $256 \times 256$ points.

Figure 2 shows the average absolute error (norm $\mathrm{L}^{1}$ ) as a function of the number of mesh cells for the different schemes after 99 iteration steps. We observe that for any given error threshold, any $\epsilon$ and any interpolation operator, the number of cells is always lower with the adaptive scheme than with the uniform one. But that does not necessarily mean that the execution time is always lower with the adaptive scheme than with the uniform one as illustrated in Fig. 3.

Figure 3 displays the average absolute error as a function of the average time to compute one iteration step (in seconds). We notice that, for the Hermite operator, the performance of the adaptive scheme is always better than the performance of the uniform scheme. But this is not the case for the Lagrange operator.

Figure 4 shows the error as a function of the number of steps for each of the two operators. We observe that the Hermite operator for $J=9$ and $\varepsilon=10^{-8}$ has a slightly better accuracy than the uniform Lagrange operator for $J=10$. Moreover, we notice that the amplitude of the error oscillation is lower for the Hermite operator than for the Lagrange operator. The execution time is also better: 0.419 seconds per iteration step with 86092 cells for the Hermite operator and 0.646 seconds with 1048576 cells for the uniform Lagrange operator.
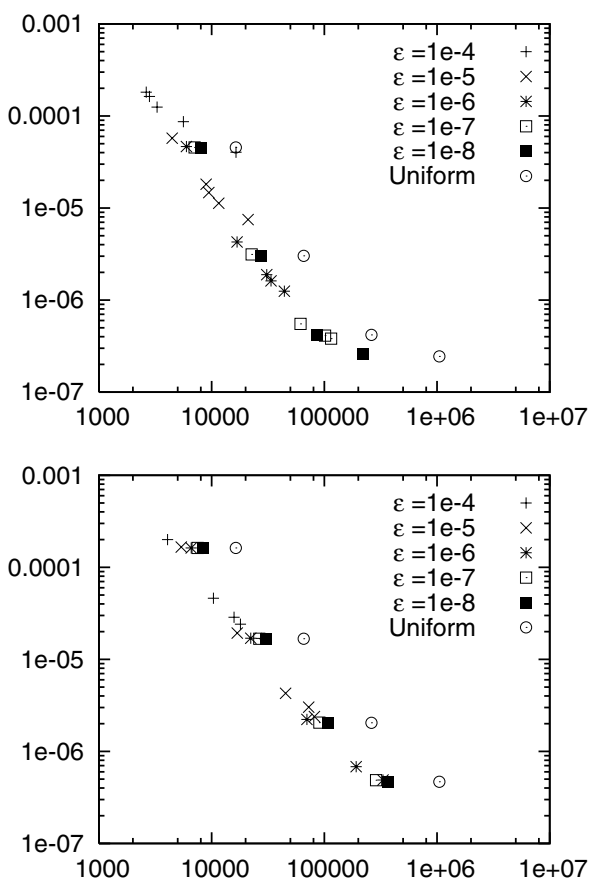

Fig. 2. Error/number of cells for Hermite (top) and Lagrange (bottom) interpolations. Each point corresponds to a value of $J$ between 7 and 11. The first three symbols go from 7 to 11, and the last three symbols go from 7 to 10 (from left to right).
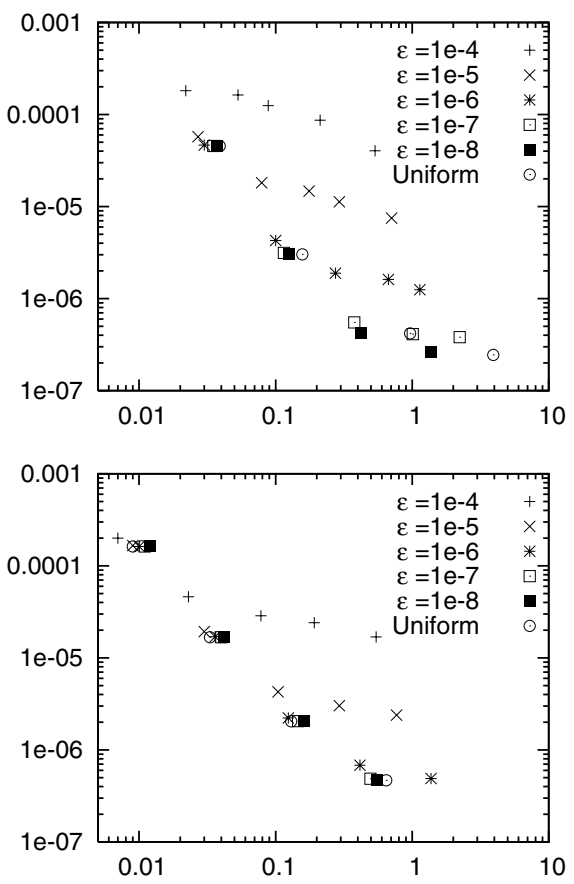

Fig. 3. Error/average time to compute one iteration step [s] for Hermite (top) and Lagrange (bottom) interpolations. Each point corresponds to a value of $J$ between 7 and 11. The first three symbols go from 7 to 11 , and the last three symbols go from 7 to 10 (from left to right). 

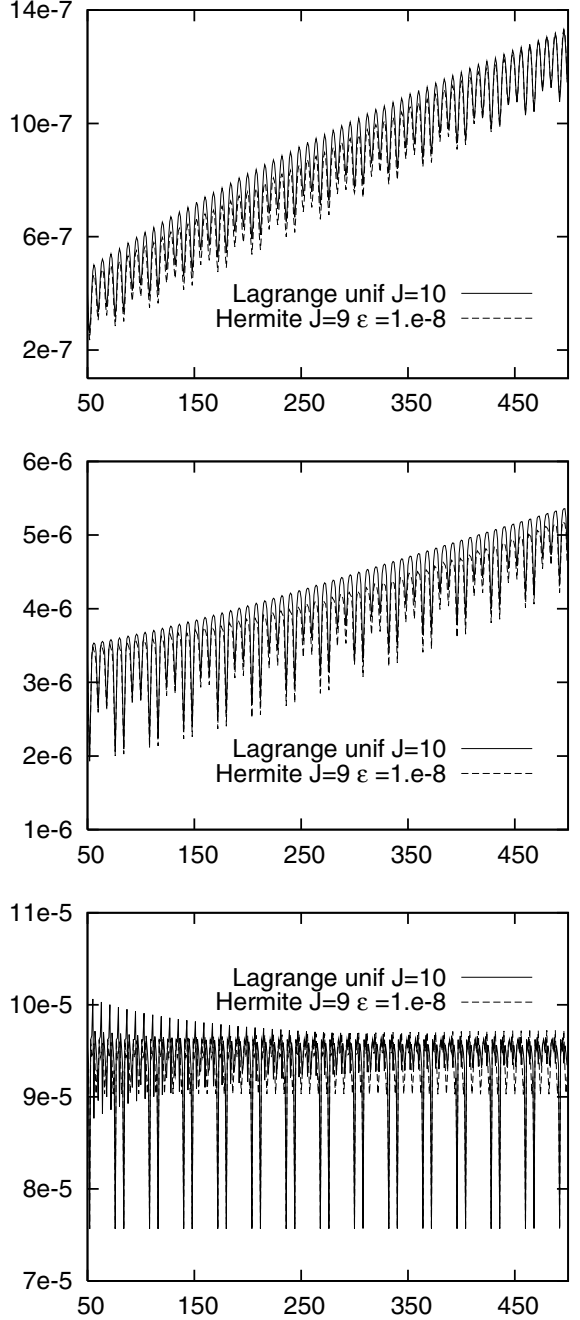

Fig. 4. Error in the norm $\mathrm{L}^{1}$ (top), $\mathrm{L}^{2}$ (middle), $\mathrm{L}^{\infty}$ (bottom) for an adaptive Hermite scheme with $J=9$ and $\epsilon=10^{-8}$, a uniform Lagrange scheme with $J=10$ and from 50 to 500 iteration steps.

\section{Conclusions}

In this paper, we defined a Hermite interpolating operator which can be used in both uniform and adaptive schemes for solving the Vlasov equation. The numerical results show that this operator offers great advantages in comparison with the Lagrange operator which was originally used.

Our first goal was to demonstrate the feasibility of developing a Vlasov solver based on the Hermite operator. Therefore, we defined it for a $2 \mathrm{D}$ phase space and considered a test case for which the analytic solution was known. Our next goal is to run more realistic test cases and extend our approach to four dimensions.

In a physical test case, the backward operator is computed at each step from the charge density. Since the computation cost of this density is proportional to the number of cells, it means that it is even more advantageous to use the Hermite operator instead of the Lagrange one, as the results in this paper show.

Further work includes parallelizing our sequential code. We plan to reuse some parallelization techniques which resulted in good speed-up on distributed memory parallel machines for a similar numerical scheme but the Lagrange operator (Hoenen et al., 2004). In particular, we designed a specific data structure (Hoenen and Violard, 2006) which is suitable to exploit data locality coming from the local nature of these schemes and which can be advantageously reused to design a code for shared memory parallel machines using OpenMP directives.

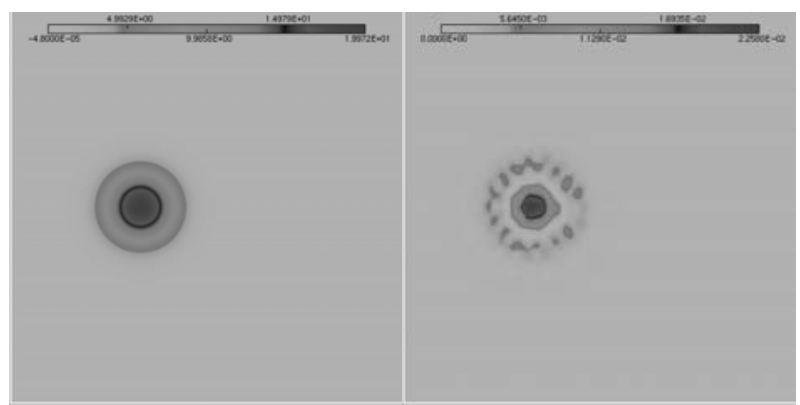

Fig. 5. Solution $f$ and the error for $\varepsilon=10^{-6}$ and $J=$ 7 after 900 steps in the Hermite case.

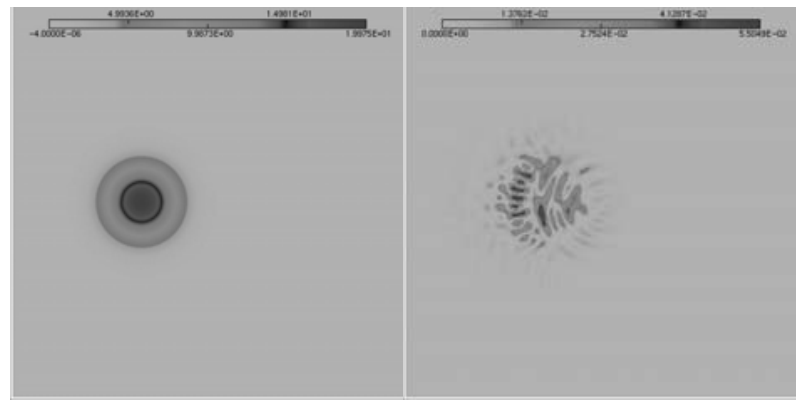

Fig. 6. Solution $f$ and the error for $\varepsilon=10^{-6}$ and $J=$ 7 after 900 steps in the Lagrange case.

\section{References}

Besse N. and Sonnendrücker E. (2003): Semi-Lagrangian schemes for the Vlasov equation on an unstructured mesh of phase space. Journal of Computational Physics, Vol. 191, No. 2, pp. 341-376.

Campos Pinto M. and Mehrenberger M. (2005): Adaptive numerical resolution of the Vlasov equation, In: Numerical Methods for Hyperbolic and Kinetic Problems (S. Cordier, T. Goudon, M. Gutnic, E. Sonnendrücker, Eds.). Zürich: European Mathematical Society, pp. 43-58.

Campos Pinto M. and Mehrenberger M. (2005): Convergence of an adaptive scheme for the one-dimensional VlasovPoisson system. Technical Report No. RR-5519, INRIA Lorraine. 
Gutnic M., Haefele M., Paun I., Sonnendrücker E. (2004): Vlasov simulations on an adaptive phase-space grid. Computer Physics Communications, Vol. 164, No. 1-3, pp. 214-219.

Gutnic M., Haefele M. and Latu G. (2005): A parallel Vlasov solver using a wavelet based adaptive mesh refinement. Proc. Int. Conf. Parallel Processing, ICPP'2005, 7th Workshop High Performance Scientific and Engineering Computing, Oslo: IEEE Computer Society Press, pp. 181-188.

Hoenen O., Mehrenberger M. and Violard E. (2004): Parallelization of an adaptive Vlasov solver, Proc. 11th European PVM/MPI Users' Group Conference, EuroPVM/MPI 2004, Berlin: Springer, pp. 430-435.

Hoenen O. and Violard E. (2006): An efficient data structure for an adaptive Vlasov solver. Research Report RR 06-02, ICPS - LSIIT laboratory (CNRS UMR-7005).
Hong D., Schumaker L.L. (2004); Surface compression using a space of $C^{1}$ cubic splines with a hierarchical basis. Geometric Modelling Computing, Vol. 72, No. 1-2, pp. 79-92.

Nakamura T. and Yabe T. (1999): Cubic interpolated propagation scheme for solving the hyperdimensional VlasovPoisson equation in phase space. Computer Physics Communications, Vol. 120, No. 2-3, pp. 122-154.

Sonnendrücker E., Filbet F., Friedman A., Oudet E., Vay J. L. (2004): Vlasov simulation of beams on a moving phasespace grid. Computer Physics Communications, Vol. 164, No. 1-3, pp. 390-395. 